\title{
Exercise Motivation and Self-Efficacy Vary Among Patients with Heart Failure - An Explorative Analysis Using Data from the HF-Wii Study
}

\author{
Leonie Klompstra (D) \\ Tiny Jaarsma $\mathbb{D}^{\prime}$ \\ Anna Strömberg $\mathbb{D}^{1,2}$ \\ Lorraine S Evangelista ${ }^{3}$ \\ Martje HL van der Wal ${ }^{1,4}$ \\ On behalf of the HF-Wii \\ Study Team
}

'Department of Health, Medicine and Caring Sciences, Linköping University, Linköping, Sweden; ${ }^{2}$ Department of Cardiology, Linköping University, Linköping, Sweden; ${ }^{3}$ University of Texas Medical Branch School of Nursing, Galveston, TX, USA; ${ }^{4}$ Department of Cardiology, University Medical Center Groningen, University of Groningen, Groningen, The Netherlands
Correspondence: Leonie Klompstra Linköping University, Department of Health, Medicine and Caring Sciences, Linköping, SE 60I 74, Sweden

Tel +4611363629

Fax +46 II I 25448

Email leonie.klompstra@liu.se
Purpose: To examine profiles in patients with heart failure (HF) regarding their exercise motivation and self-efficacy.

Patients \& Methods: The baseline data of patients with HF participating in the HFWii study were analysed. In total, 517 patients were divided into four groups based on their exercise motivation (exercise motivation index) and self-efficacy (exercise selfefficacy scale). To describe the differences in demographic and clinical variables between the groups, chi-square cross-tabulations and ANOVAs were conducted.

Results: The four groups were labelled as insecure avoiders (25\%), laid-back strugglers (10\%), conscientious self-doubters (42\%) and determined achievers $(22 \%)$. Patients' profiles differ according to their motivations and self-efficacy towards exercise. Most patients were conscientious self-doubters (high motivation and low selfefficacy), and these patients had more comorbidities and lower exercise capacity compared to the other groups, which could decrease their confidence in exercising. However, only half of the patients who were determined achievers (high motivation and high self-efficacy) reached the recommended amount of physical activity per week. This indicates that motivation and self-efficacy are crucial determinants, but more factors are important for becoming more physically active.

Conclusion: Understanding patients' motivations and self-efficacy are necessary in order to provide meaningful physical activity counselling and promotion.

Keywords: motivation, self-efficacy, physical activity, exercise, heart failure

\section{Plain Language Summary}

This study examined profiles in patients with heart failure regarding their motivation and confidence towards exercise. In total, 517 patients were divided into four groups based on their exercise motivation and confidence to exercise. The four groups were labelled as insecure avoiders (25\%), laid-back strugglers (10\%), conscientious selfdoubters $(42 \%)$ and determined achievers (22\%). Patients' profiles differ according to their motivations and confidence towards exercise. Most patients were conscientious self-doubters (high motivation and low confidence), and these patients suffered from more diseases and lower exercise capacity compared to the other groups, which could decrease their confidence in exercising. However, only half of the patients who were determined achievers (high motivation and high confidence) reached the recommended amount of physical activity per week (150 min exercise a week). This indicates that motivation and confidence are crucial determinants, but more factors are important for becoming more physically active. 


\section{Introduction}

The health benefits of regular exercise in patients with heart failure (HF) are widely recognized, and exercise training is now a well-established therapy for patients with $\mathrm{HF}^{1,2}$ Regular exercise is safe, can decrease mortality, ${ }^{3,4}$ and can improve exercise capacity and quality of life. ${ }^{5,6}$ Despite the benefits and strong recommendations in the ESC guidelines, ${ }^{7}$ adherence to exercise recommendations is low (39\%), and is lower than most other selfmanagement activities such as adherence to diet $(83 \%)$ or medication (higher than 90\%).,

There is growing evidence that crucial determinants of exercise adherence are self-efficacy and motivation. Selfefficacy refers to the belief in one's ability to execute tasks in a given situation. ${ }^{10,11}$ Self-efficacy is related to overcoming barriers to exercise, and has been specifically linked to exercise adherence and maintenance in patients with HF. ${ }^{12-15}$ Barriers to exercise can be patient-related (eg, inadequate social support), social and economic factors, barriers in the health care team system (eg, only $10 \%$ of eligible HF patients receive a cardiac rehabilitation referral), condition-related factors (eg, experience of symptoms) or therapy-related factors (eg, enjoyment of exercise). ${ }^{16-19}$ A lack of motivation can also be a barrier, and reduces the amount of exercise and exercise adherence in patients with HF. ${ }^{15,20-22}$ Motivation is defined as the intrinsic determination towards goal attainment. ${ }^{23}$ Selfefficacy is also known to mediate the relationship between motivation and exercise. ${ }^{15,24}$ This means that even if a patient is motivated to exercise, the patient will not exercise if they do not feel confident about exercising. As self-efficacy and motivation influence each other and the amount of exercise in patients with HF, giving patientcentred care and personalizing exercise recommendations should be assessed.

We hypothesize that patients' profiles differ in terms of motivation and self-efficacy. If profiles differ, these patients should receive different exercise advice or exercise help. Therefore, the aim of this study was to examine patient profiles regarding their exercise motivation and self-efficacy.

\section{Methods}

The baseline data of patients with HF participating in the HF-Wii study ${ }^{25}$ were analysed. The HF-Wii study is a physical activity trial assessing the effects of exergaming on submaximal exercise capacity. Patients were eligible for this study if they were diagnosed with HF, were older than 18 years of age, had no problems with mobility, balance or sight, or a severe cognitive dysfunction that could prohibit them from exergaming, had an estimated survival chance of more than six months, and did not have difficulties understanding or reading the language where this intervention took place. All patients provided informed consent.

The study was conducted according to the principles of the Declaration of Helsinki (2008) in accordance with the Medical Research Involving Human Subjects Act. In Sweden, ethical approval was obtained centrally (Etikprövningsmyndigheten DNR 2012/247-31). Additional approval was obtained from local review boards (the Netherlands: Ethics Review Committee azM/ UM NL48647.068.14/METC141085; Italy: Comitato Etico Lazio 2 0052838/272/UVF/1; Israel: Institutional Review Board Rabin medical center 0022-13-RMC; Germany: Ethikkomission der Landesärztekammer Brandenburg S22(a)/2015; USA Institutional Review Board University of California, Irvine UCI IRB HS\# 2016-2955). The trial was registered in ClinicalTrials.gov (NCT01785121).

\section{Measurements}

Motivations were measured using the Exercise Motivation Index, ${ }^{26}$ including 15 motivations with answers ranging from 0 (not important) to 4 (extremely important), with three subscales (physical, psychological and social motivations).

Self-efficacy was measured using the Exercise SelfEfficacy Scale, ${ }^{27}$ including six possible barriers, with answers ranging from 1 (not confident about overcoming the barrier) to 10 (very confident about overcoming the barrier).

Self-reported physical activity was assessed with a single question:

Over the past week (even if it was not a typical week), how much time did you spend exercising or being physically active (e.g., strength training, walking, swimming, gardening or other type of training)?

With five possible answers: (1) No time, (2) Less than 30 minutes, (3) 30-60 minutes, (4) 1-3 hours and (5) More than three hours. More than three hours a week was recorded as physically active.

Additional data were collected on submaximal exercise capacity (six-minute walk test), symptoms of dyspnoea and fatigue (measured on a numeric rating scale from $0-10$, with 0 meaning "not experienced any symptoms" and ten 
meaning "experienced worst possible symptoms"), anxiety and depression (Hospital Anxiety and Depression Scale, ${ }^{28}$ with a score of 7 or higher being seen as having depressive or anxiety symptoms), sleep (the Minimal Insomnia Symptom Scale, ${ }^{29}$ with a cut-off score of six or higher on the scale being seen as having sleeping problems), cognition (Montreal Cognitive Assessment, ${ }^{30}$ where a score of 18-25 was seen as having mild cognitive impairment, 10-17 as moderate cognitive impairment and less than ten as severe cognitive impairment), health-related quality of life (Minnesota Living with Heart Failure Questionnaire, ${ }^{31}$ where the total score could range from zero to 105 , with higher scores indicating more significant impairment on health-related quality of life) and well-being (Cantril's ladder of life, ${ }^{32}$ measured on a numeric rating scale from $0-10$ ).

\section{Statistical Analyses}

Only patients who had completed the measurements for exercise motivation and self-efficacy (517 of 605) were included in the current analysis. For presentation reasons, we dichotomized the total score for exercise self-efficacy. A total score below six was seen as low confidence in exercising, whereas a total score of equal to or higher than six was seen as having the confidence to exercise. The total score from the Exercise Motivation Index was also dichotomized. A total score lower than two was seen as low motivation to exercise, while a score of two or higher was seen as having the motivation to exercise.
Patients were divided into four groups. Insecure avoiders were those patients with low motivation and low confidence in exercising; laid-back strugglers were patients with low motivation but who had the confidence to exercise; conscientious self-doubters were patients who were motivated but had low confidence in exercising; and determined achievers were patients who were motivated and had the confidence to exercise (Figure 1).

The patients' profiles in each group were examined based on demographics and clinical characteristics, physical activity and exercise, symptoms, anxiety, depression, sleep, cognition, health-related quality of life, and well-being.

To describe the difference between the four groups in terms of motivation and self-efficacy, chi-square crosstabulations and ANOVAs were conducted on the variables listed above.

When significant differences were observed between the groups, paired group differences were examined with post-hoc (Bonferroni) analyses with continuous variables. With categorical data, ChiSquare analyses were carried out to look at the differences between the paired groups. A p-value $<0.008$ was seen as significantly different, as corrected for six different comparisons (Supplement 1).

\section{Results}

The included 517 patients with HF had a mean age of 67 $( \pm 12) .71 \%$ were men $(n=342)$, and most of the patients were married or in a relationship $(n=371,72 \%)($ Table 1$)$.

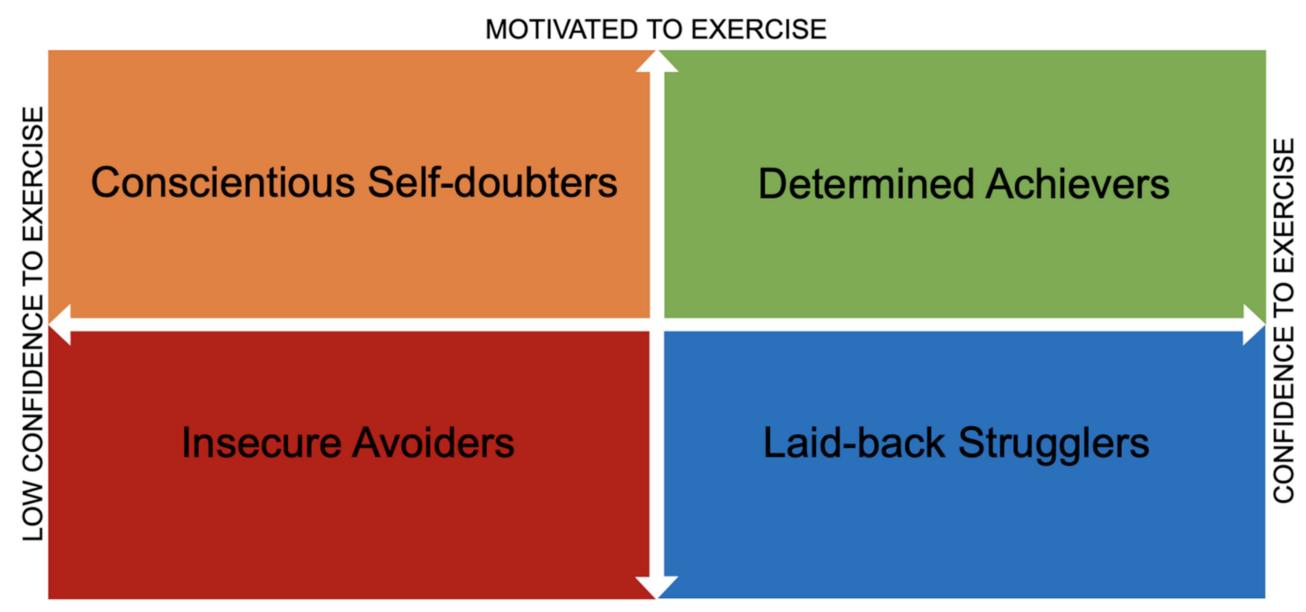

LOW MOTIVATION TO EXERCISE

Figure I Four groups based on their level of exercise motivation and exercise self-efficacy. Insecure avoiders are patients with low motivation and low confidence in exercising; laid-back strugglers are those with low motivation and confidence to exercise; conscientious self-doubters are those motivated but with low confidence in exercising; and determined achievers are those motivated and with confidence to exercise. 
Most patients were in New York Heart Association (NYHA) class I or II $(69 \%, \mathrm{n}=405)$. There were no significant differences between the 517 patients included in this study and the 88 patients who were excluded due to incomplete values regarding age, gender or NYHA class.

\section{Exercise Motivation}

In the group as a whole, the mean motivation per item was $2.32 \pm 0.88$ and physical and psychological motivations were rated as the most important $(2.66 \pm 0.88$ and 2.51 \pm 0.92 ) (Table 2).

The most important physical motivation was "I want to be healthier and perhaps live longer" ( $80 \%$ of the patients rated this as important/very important), and the most important psychological motivation was "exercise increases my overall sense of well-being" (63\% rated this as important/very important). However, social motivations were rated as the least important (mean 1.79 \pm 1.10 ), and 39\% of the patients found it important "to be as active as my family and friends".

\section{Exercise Self-Efficacy}

The mean total score for exercise self-efficacy was 5.02 $( \pm 1.98)$ (Table 2).

For every barrier on the self-efficacy scale, less than $50 \%$ of the patients were confident about overcoming them. Experienced barriers that were the most difficult to overcome were feeling physically fatigued $(n=173,34 \%)$ and taking time for the family $(n=203,39 \%)$.

\section{Descriptions of Patients' Profiles Regarding Motivation and Confidence in Exercising}

Patients' profiles were assessed regarding their exercise motivation and exercise self-efficacy (Figure 1). Table 1 reports the differences in demographic and clinical characteristics between these patient groups. Table 2 reports the differences in exercise motivation and confidence in exercising. The most notable differences in patient profiles between the groups are described for each group.

\section{Insecure Avoiders: Low Exercise Motivation and Low Confidence}

In this group, patients had low motivation (mean motivation was $1.38 \pm 0.42$ ) and low confidence (a mean selfefficacy score of $3.71 \pm 1.30$ ). This group represents $25 \%$ $(n=130)$ of patients included in this study.

Patients in this group had low self-reported levels of physical activity, and only $26 \%$ exercised for more than three hours a week. They had a high exercise capacity, as these patients walked 416 metres $( \pm 132)$, which was significantly higher than the conscientious self-doubters (Table 1).

Patients in this group also reported low well-being (5.91 \pm 2.03), which was significantly lower than patients who were motivated and were confident about exercising (group 4).

\section{Laid-Back Strugglers: Low Exercise Motivation, but Confident}

In this group, patients had low motivation (mean motivation was $1.50 \pm 0.34$ ) but were confident about exercising (mean self-efficacy was 7.28 \pm 1.04 ). This group represented $10 \%(n=54)$ of the patients included in this study.

Forty percent of the patients in this group exercised for more than three hours a week. They had the highest exercise capacity compared to the other groups, walking 472 metres $( \pm 100)$, which was significantly higher than the conscientious self-doubters (Table 1).

This group of patients was significantly younger $(46 \%$ $\geq 65$ years old) than all the other groups, and were more often in NYHA class I or II (16\%).

The group had the lowest number of comorbidities, such as chronic obstructive pulmonary disease (COPD) (11\%) and depression (13\%), and reported a low amount of anxiety (15\%) and sleeping problems (12\%). However, they reported the highest score for shortness of breath $(5.13 \pm 2.18)$. They also reported the highest quality of life scores $(24.70 \pm 18.87)$, both physically and emotionally.

\section{Conscientious Self-Doubters: Motivated to Exercise, but Low Confidence}

In this group, patients were motivated to exercise (mean motivation was $2.84 \pm 0.61$ ) but had low confidence (mean self-efficacy was $4.04 \pm 1.24$ ). This group represents $42 \%$ $(n=217)$ of the patients included in this study.

In this group, $39 \%$ of the patients exercised for more than three hours a week, and their exercise capacity was the lowest $(359 \pm 157$ metres in the six-minute walk test).

Patients in this group were significantly older $(69 \%$ $\geq 65$ years old) and more often classified as NYHA III or IV $(39 \%)$ than the other groups. One-third of the patients in this group suffered from depressive symptoms, $42 \%$ suffered from anxiety, 32\% experienced sleeping problems and $25 \%$ had COPD. The patients in this group had the lowest quality of life $(41.75 \pm 22.29)$, in both the emotional and physical dimensions, and reported the lowest well-being $(5.87 \pm 1.95)$. 


\begin{tabular}{|c|c|c|c|c|c|c|c|c|c|c|}
\hline$m s$ & & & & & $*$ & & & & $*$ & \\
\hline$N \stackrel{n}{s}$ & & & & & & & * & & & \\
\hline$N \stackrel{n}{ }$ & $*$ & & & & $*$ & & * & & * & \\
\hline$-s \sigma$ & & & & & & & & & & \\
\hline$-\stackrel{n}{>} m$ & & & & & $*$ & & & & $*$ & \\
\hline$-\sum N$ & & & & & & & $*$ & & & \\
\hline$\stackrel{0}{5}_{i=0}^{5}$ & $\bar{j}$ & $\frac{n}{0}$ & $\frac{\pi}{\overline{0}}$ & 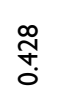 & ō & $\frac{\text { g }}{0}$ & 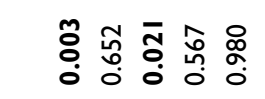 & m. & ¿্口 & 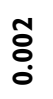 \\
\hline 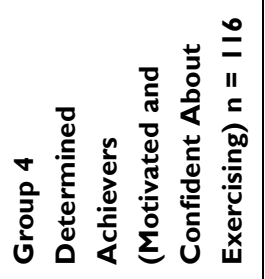 & $\begin{array}{l}2 \\
+1 \\
\hat{+1}\end{array}$ & 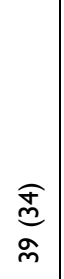 & 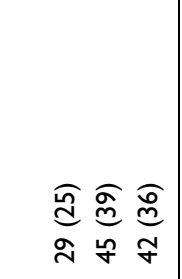 & 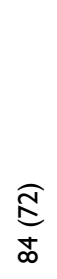 & 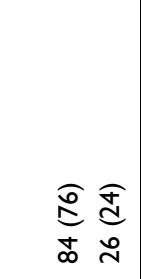 & 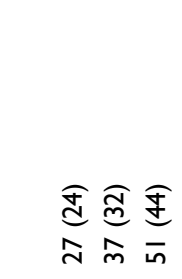 & 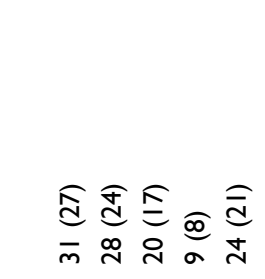 & $\begin{array}{l}\underset{i}{1} \\
\underline{\omega} \\
\underline{n}\end{array}$ & $\begin{array}{l}\stackrel{\llcorner}{m} \\
\stackrel{+1}{+} \\
\stackrel{+}{\sigma}\end{array}$ & $\begin{array}{l}\text { व్} \\
\text { क्र } \\
\text { సे }\end{array}$ \\
\hline 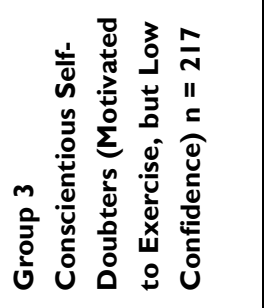 & $\begin{array}{l}\simeq \\
\simeq+1 \\
\infty \\
0\end{array}$ & 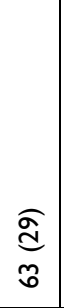 & 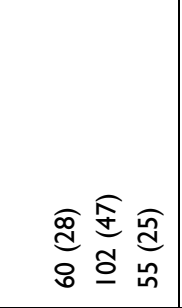 & $\begin{array}{l}\widehat{\sigma} \\
\hat{\sigma} \\
\stackrel{\circ}{0}\end{array}$ & $\begin{array}{ll}\widehat{\bar{\theta}} & \widehat{\sigma} \\
0 & \stackrel{e}{c} \\
\underline{m} & \infty \\
\infty\end{array}$ & 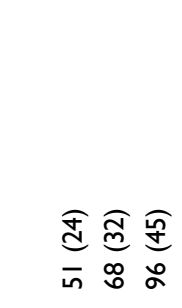 & 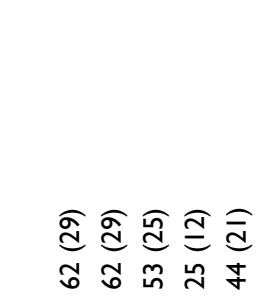 & 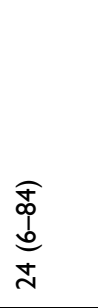 & $\begin{array}{l}\frac{n}{n} \\
+1 \\
o \\
m\end{array}$ & 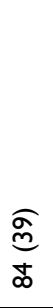 \\
\hline 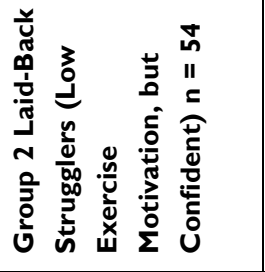 & $\begin{array}{l}a \\
+1 \\
0\end{array}$ & $\stackrel{E}{E}$ & 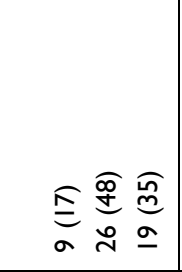 & 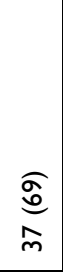 & $\begin{array}{ll}\underset{\infty}{\infty} & \widehat{\sigma} \\
\mathcal{f} & \stackrel{\infty}{=}\end{array}$ & 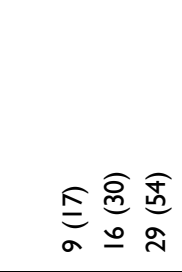 & 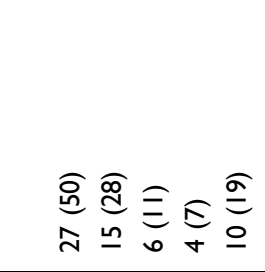 & $\begin{array}{l}\widehat{F} \\
\vdots \\
\infty \\
\varrho \\
\underline{0}\end{array}$ & 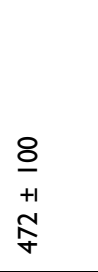 & $\frac{\bar{o}}{\bar{i}}$ \\
\hline 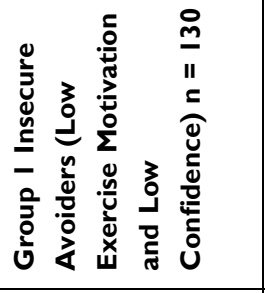 & $\begin{array}{l}2 \\
+1 \\
6 \\
\end{array}$ & 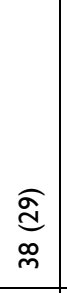 & 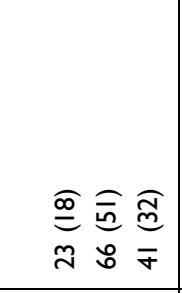 & $\begin{array}{l}E \\
\stackrel{8}{0} \\
\end{array}$ & 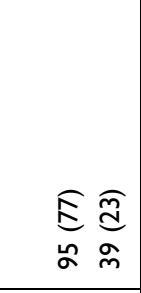 & 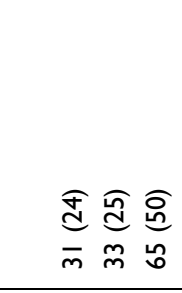 & 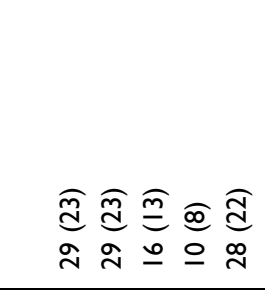 & $\begin{array}{l}\widehat{\tilde{p}} \\
\hat{b} \\
\infty \\
o\end{array}$ & $\begin{array}{l}\stackrel{N}{m} \\
+1 \\
\stackrel{0}{+}\end{array}$ & 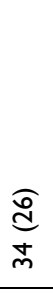 \\
\hline $\begin{array}{l}\text { in } \\
\text { II } \\
\frac{5}{5} \\
\stackrel{0}{\circ}\end{array}$ & $\begin{array}{l}2 \\
+1 \\
6\end{array}$ & $\begin{array}{l}\underset{\Xi}{d} \\
\underline{\underline{N}}\end{array}$ & 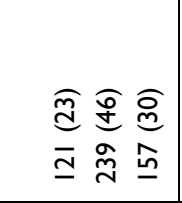 & $\begin{array}{l}\widehat{E} \\
\mathbb{E} \\
m\end{array}$ & 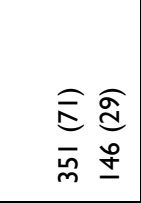 & 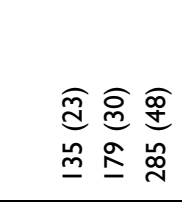 & 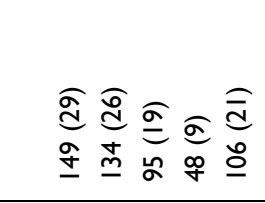 & 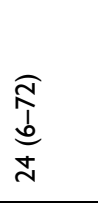 & $\begin{array}{l}\frac{u}{q} \\
+1 \\
\sigma \\
\sigma\end{array}$ & 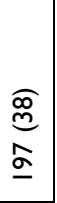 \\
\hline & 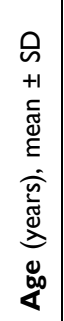 & 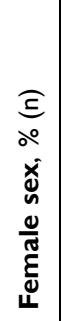 & 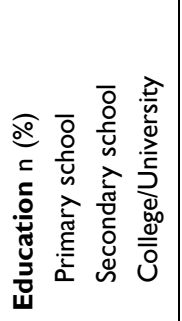 & 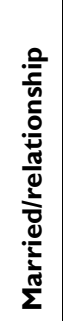 & 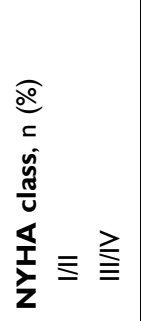 & 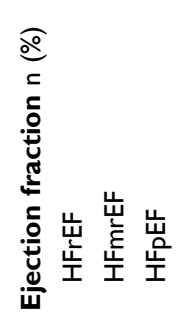 & 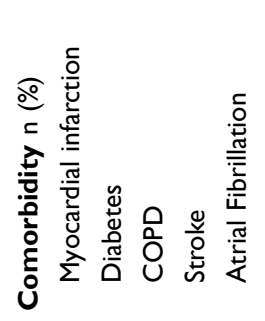 & 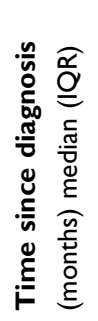 & 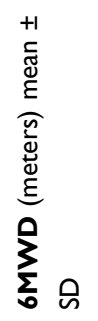 & 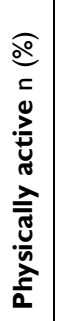 \\
\hline
\end{tabular}




\begin{tabular}{|c|c|c|c|c|c|c|c|c|}
\hline$m>r$ & & & * & * & & $*$ & $*$ & \multirow{13}{*}{ 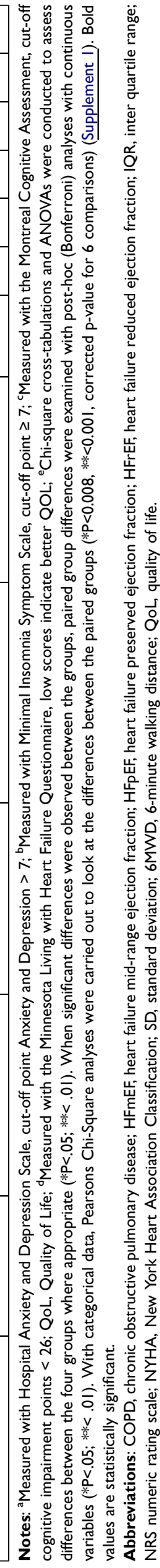 } \\
\hline$N \Sigma$ & & & & & & & & \\
\hline$N>m$ & & * & $*$ & $*$ & & $* *$ & * & \\
\hline$-n \sigma$ & * & & & & & & $*$ & \\
\hline$-n m$ & & $*$ & & & $*$ & $* *$ & & \\
\hline$-\sum n$ & & & & & & & & \\
\hline 品 & $\begin{array}{l}\text { ồ } \\
\text { o̊ } \\
0\end{array}$ & $\begin{array}{l}\overline{8} \\
\dot{0}\end{array}$ & '̄ & 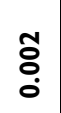 & $\begin{array}{l}m \\
0 \\
0 \\
0\end{array}$ & 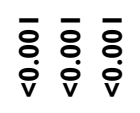 & $\begin{array}{l}\overline{8} \\
\dot{i}\end{array}$ & \\
\hline 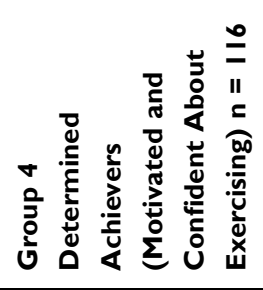 & \begin{tabular}{ll}
$\infty$ & 0 \\
$i$ & $\stackrel{+}{+}$ \\
+1 & +1 \\
\hdashline & + \\
+
\end{tabular} & 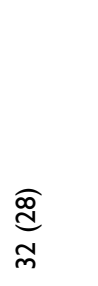 & $\underset{\underline{\infty}}{\stackrel{0}{\sigma}}$ & $\frac{\underset{\infty}{=}}{\bar{N}}$ & $\begin{array}{l}a \\
0 \\
a \\
0\end{array}$ & 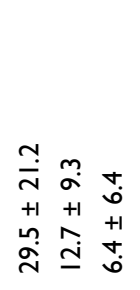 & $\begin{array}{l}n \\
i \\
+1 \\
o \\
i\end{array}$ & \\
\hline 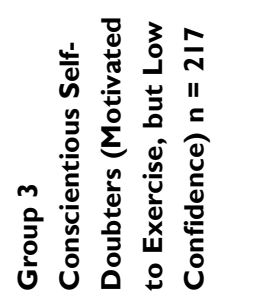 & 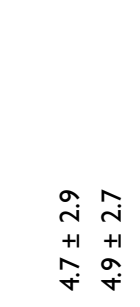 & $\begin{array}{l}\widehat{I} \\
\sigma\end{array}$ & 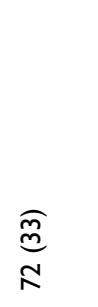 & $\begin{array}{l}\text { శ్} \\
0 \\
0\end{array}$ & 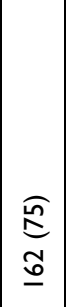 & 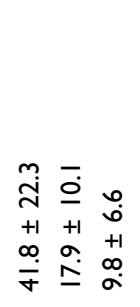 & $\begin{array}{l}\stackrel{0}{i} \\
++ \\
\dot{+} \\
\dot{\rho}\end{array}$ & \\
\hline 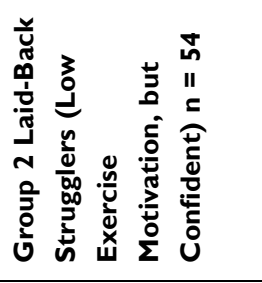 & 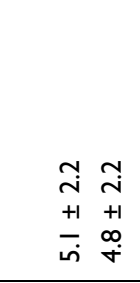 & $\underset{\infty}{\stackrel{\Omega}{n}}$ & $\stackrel{\widehat{m}}{\stackrel{n}{n}}$ & $\underset{\Xi}{\Xi}$ & $\begin{array}{l}\text { E } \\
\frac{n}{m}\end{array}$ & 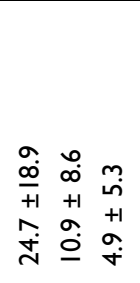 & $\begin{array}{l}\stackrel{\infty}{+} \\
\stackrel{+}{+1} \\
\hat{0}\end{array}$ & \\
\hline 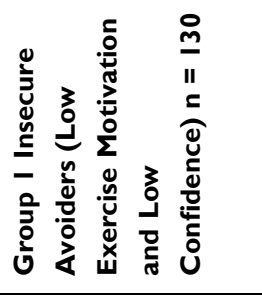 & 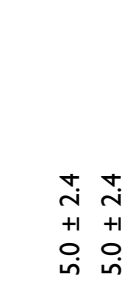 & 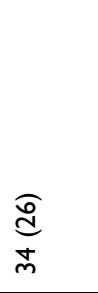 & $\begin{array}{l}\widehat{\widetilde{d}} \\
\stackrel{0}{0}\end{array}$ & $\begin{array}{l}\widehat{\bar{d}} \\
\bar{\lambda}\end{array}$ & 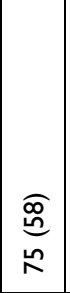 & 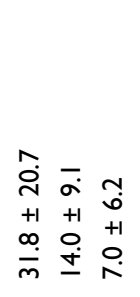 & $\begin{array}{l}\dot{i} \\
+1 \\
a \\
\dot{m}\end{array}$ & \\
\hline \multirow[t]{2}{*}{ 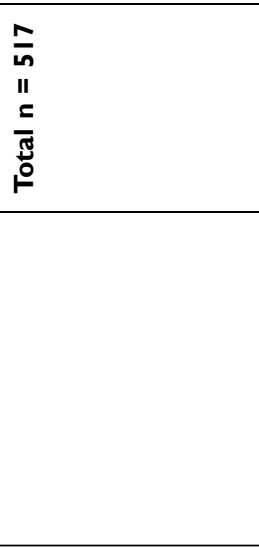 } & $\begin{array}{ll}\hat{i} & \hat{i} \\
+1 & +1 \\
\hat{+} & \stackrel{+}{+}\end{array}$ & $\begin{array}{l}\text { } \\
\stackrel{0}{n} \\
\underline{\underline{0}}\end{array}$ & 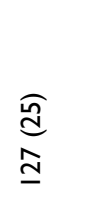 & 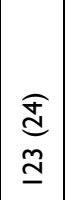 & 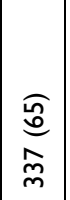 & 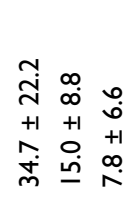 & \begin{tabular}{l}
$\bar{i}$ \\
+1 \\
\multirow{H}{*}{}
\end{tabular} & \\
\hline & 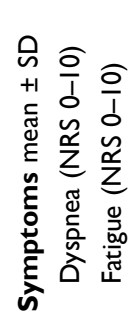 & 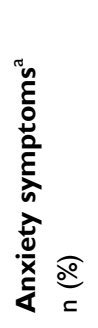 & 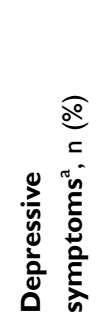 & 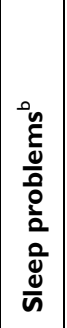 & 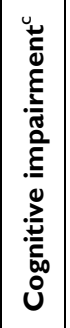 & 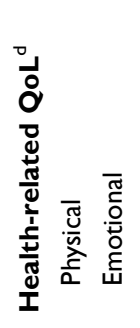 & 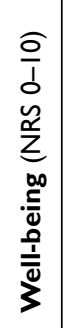 & \\
\hline
\end{tabular}


Table 2 Description of Motivation and Confidence to Exercise in the Whole Group $(n=517)$ and the Four Profile Groups

\begin{tabular}{|c|c|c|c|c|c|}
\hline & Total $n=517$ & $\begin{array}{l}\text { Insecure Avoiders } \\
n=130\end{array}$ & $\begin{array}{l}\text { Laid-Back } \\
\text { Strugglers } n=54\end{array}$ & $\begin{array}{l}\text { Conscientious Self- } \\
\text { Doubters } n=217\end{array}$ & $\begin{array}{l}\text { Determined } \\
\text { Achievers } n=116\end{array}$ \\
\hline Exercise motivation (Range $0-4$ ) mean $\pm S D$ & $2.32 \pm 0.88$ & $1.38 \pm 0.42$ & $1.50 \pm 0.34$ & $2.84 \pm 0.61$ & $2.88 \pm 0.60$ \\
\hline Physical motivation (Range $0-4$ ) mean $\pm S D$ & $2.66 \pm 0.88$ & $1.78 \pm 0.89$ & $1.84 \pm 0.44$ & $3.15 \pm 0.60$ & $3.24 \pm 0.58$ \\
\hline Social motivation (Range $0-4$ ) mean \pm SD & $1.79 \pm 1.10$ & $0.80 \pm 0.53$ & $0.83 \pm 0.54$ & $2.38 \pm 0.90$ & $2.32 \pm 1.01$ \\
\hline Psychological motivation (Range 0-4) mean \pm SD & $2.51 \pm 0.92$ & $1.56 \pm 0.61$ & $1.82 \pm 0.56$ & $2.98 \pm 0.66$ & $3.09 \pm 0.64$ \\
\hline Main total self-efficacy (Range $1-10$ ) mean $\pm S D$ & $5.02 \pm 1.98$ & $3.71 \pm 1.30$ & $7.28 \pm 1.04$ & $4.04 \pm 1.24$ & $7.29 \pm 1.00$ \\
\hline
\end{tabular}

Abbreviation: SD, standard deviation.

\section{Determined Achievers: Motivated and Confident About Exercising}

In this group, patients were both motivated (mean motivation $2.88 \pm 0.60$ ) and confident about exercising (mean selfefficacy was $7.29 \pm 1.00$ ). This group represents $22 \%$ $(\mathrm{n}=116)$ of the patients included in this study.

This group had the highest number of patients who were physically active, with half of the patients exercising for more than three hours a week. Patients in this group had a high exercise capacity, walking a mean of 424 $( \pm 135)$ metres in the six-minute walk test.

They reported the highest well-being $(7.03 \pm 1.67)$ and the lowest score for shortness of breath $(4.05 \pm 2.79)$.

\section{Discussion}

To our knowledge, this is the first study to examine the profiles of patients with heart failure based on their exercise motivation and self-efficacy.

Understanding patients' motivations and self-efficacy is important in order to provide effective physical activity counselling and promotion. This study shows that patients' profiles vary in terms of motivation and self-efficacy (confidence) to exercise. Many patients (64\%) were motivated to become more physically active, and one-third were confident about exercising.

Most patients in this study ( $\mathrm{n}=217,36 \%)$ were highly motivated, but did not have the confidence to overcome barriers to exercise (conscientious self-doubters). These patients had a low exercise capacity, and only $40 \%$ were physically active (more than three hours a week, moderate to vigorous, physically active). Previous research has shown that for patients who are highly motivated but have no confidence in their ability to overcome barriers, it is unlikely that they will increase their physical activity. ${ }^{15}$

These findings imply that instead of mainly focusing on motivating patients to exercise, the first step should be to identify barriers to exercise and build confidence to tackle these barriers. Strategies that have been described as increasing self-efficacy in patients with HF are performing exercise gradually and graded mastery, applying vicarious experience (directly observing one's own performance or someone else's performance), providing feedback and persuasion for physical activity, symptom assessment, education, recognition and reinterpretation of HF symptoms, and problem-solving regarding barriers to physical activity. ${ }^{33,34}$

Patients who were motivated to exercise but had low confidence (conscientious self-doubters) suffered from depressive symptoms and sleeping problems, decreasing their physical activity. ${ }^{35}$ Treatment for HF comorbidities, such as sleeping problems, diabetes mellitus, chronic kidney failure and depressive symptoms, will be necessary for patients' exercise capacity and quality of life. ${ }^{36}$ Still, $60 \%$ of these patients were in NYHA class I or II, which means that they experienced no HF symptoms or only mild HF symptoms while exercising. Including caregivers in future interventions to increase social support for exercise could enhance patients' confidence in exercising. ${ }^{37,38}$ Studies show that patients could be inspired by others (eg, other patients, friends or family) who were, or encouraged them to be, physically active. ${ }^{39-41}$ They expressed that being physically active in a group made it easier to exercise, but having no social support made it difficult to participate in exercise. ${ }^{39}$ This highlights the importance of the possibility for patients to include caregivers or exercise in a group to increase their confidence to exercise.

The patients who had low motivation to exercise but were confident about exercising (laid-back strugglers) reported the highest exercise capacity. However, only $39 \%$ reported being physically active for more than three hours a week. Compared with the other groups, the patients in this group were the youngest. Another study ${ }^{40}$ confirms age differences in outcomes of cardiac rehabilitation programs. They found that middle-aged patients experience more improvement in physiologic as older- 
aged patients in the same cardiac rehabilitation program, suggesting that physical activity advice and cardiac rehabilitation should be age adapted. The laid-back strugglers also had the highest quality of life and well-being and the lowest symptoms of depression and anxiety. In motivating patients to become more physically active, the first step is to get them interested in physical activity. Our study showed that motivation might be different, and a general approach, such as "exercise is good for everybody", will not increase physical activity. A patient can be motivated to avoid cardiovascular disease but not be interested in a physical activity intervention. ${ }^{42}$ Behavioural experience and self-regulation (goal setting, action planning, selfmonitoring of behaviour, feedback on behaviour and problem-solving) are strategies to increase motivation and positive changes in physical activity. ${ }^{43,44}$

Our findings showed that only one in five patients were motivated and confident about exercising (determined achievers). Among those patients with high motivation and high self-efficacy, only half were physically active for more than three hours a week. Factors other than motivation and selfefficacy are important for becoming more physically active. Patients might have experienced disease-specific barriers, such as side effects of medication or HF symptoms that prevent them from being more physically active. A lack of social support could be one explanation for not becoming more physically active. ${ }^{19}$ Offering these patients physical activity programmes where care-givers are included or physical activity programmes with other patients, for example within patient organizations, could increase social support.

One out of four patients in our study had low motivation and low self-efficacy (insecure avoiders). It is important to provide a broad intervention that includes both motivation and self-efficacy strategies, as only one-third of the patients in this group were physically active.

The results of this study can be used to tailor interventions to specific subgroups of patients. Various interventions studying enhancing exercise participation have consistently revealed limited evidence of long-term effects for patients with HF. Unfortunately, patients with the most to gain from exercise activity (especially older patients with more symptoms) are least likely to participate. ${ }^{44,45}$ A major challenge in physical activity counselling and promotion is to provide clear feedback to individuals with personalized and meaningful information that motivates individuals to increase or sustain their physical activity. ${ }^{46}$

It is important to have a meaning-centred existential perspective when advising cardiac patients about their physical activities, starting from their perceptions and exercise experiences concerning their heart disease. ${ }^{47}$

One limitation of this study was that most of the patients were in NYHA class I or II (71\%), and the results could only apply to patients with low symptom experience during physical activity. This could be due to the fact that this research only included patients with HF living at home.

\section{Conclusions}

Patient profiles differ according to their motivations and self-efficacy towards exercise. Most patients were motivated but did not have the confidence to become more physically active. These patients had more comorbidities and low exercise capacity, which could decrease their confidence in exercising. However, even among those patients who were motivated and confident about exercising, only half reached the recommended amount of weekly physical activity. This indicates that factors other than motivation and self-efficacy are also important in terms of becoming more physically active.

\section{Data Sharing Statement}

The datasets used and/or analyzed during the current study are available from the corresponding author on reasonable request.

\section{Acknowledgments}

This article is written on behalf of the HF-Wii study team: T. Jaarsma, A. Strömberg, L. Klompstra, B. Ben Avraham, T. Ben Gal, J. Boyne, K. Dickstein, M. Bäck, O. Chiala, L. Evangelista, A. Hagenow, A. Hoes, E. Hägglund, J. Mårtensson, M.F. Piepoli, E. Vellone and N.P.A. Zuithoff.

We also want to thank: Norrköping: A. Hammarskiold, L. Nestor, C. Norrman, M. Viklander, A. Waldemar, R. M. Petterson; M. Wärfman. Jönköping: E. Lundberg, H. Sköldbäck, M. Sahlin.

Linköping: A. Gylling, L. Hjelmfors, M. Huss, M. Jonsson, N.P. Kato, P. Wodlin.

Stockholm: E. Hägglund, U. Lennmark.

Nyköping: E. Säfström.

The Netherlands: H.P. Brunner-La Rocca, M. Spanjers,

A. van de Voorde, G. Cleuren.

Italy: R. Corsi, G.A. Ortali,

Israel: S. Donanhirsh, Y. Navon, V. Yaari.

Germany: A. Kuntzsch.

USA: J. Ardo, J. Nguyen, M. Cacciata.

Statistical advice: M. Fredrikson, N.P.A. Zuithoff. 


\section{Author Contributions}

All authors made a significant contribution to the work reported, whether that is in the conception, study design, execution, acquisition of data, analysis and interpretation, or in all these areas; took part in drafting, revising or critically reviewing the article; gave final approval of the version to be published; have agreed on the journal to which the article has been submitted; and agree to be accountable for all aspects of the work.

\section{Funding}

This work was supported by the Swedish National Science Council (K2013-69X-22302-01-3, 2016-01390), the Swedish National Science Council/the Swedish Research Council for Health, Working Life and Welfare, VRFORTE (2014-4100), the Swedish Heart and Lung Association (E085/12), the Swedish Heart and Lung Foundation (20130340, 20160439), the Vårdal Foundation (2014-0018) and the Medical Research Council of Southeast Sweden (FORSS 474681).

\section{Disclosure}

The authors report no conflicts of interest in this work.

\section{References}

1. Pelliccia A, Sharma S, Gati S, et al. 2020 ESC Guidelines on sports cardiology and exercise in patients with cardiovascular diseaseThe Task Force on sports cardiology and exercise in patients with cardiovascular disease of the European Society of Cardiology (ESC). Eur Heart J. 2021;42(1):17-96.

2. Arena RA. Functional capacity and exercise training have earned a primary role in the assessment and treatment of patients with heart failure. Heart Fail Clin. 2015;11:xv-xvii. doi:10.1016/j.hfc.2014.10.001

3. Forman DE, Sanderson BK, Josephson RA, et al. Heart failure as a newly approved diagnosis for cardiac rehabilitation: challenges and opportunities. J Am Coll Cardiol. 2015;65(24):2652-2659. doi:10.1016/j.jacc.2015.04.052

4. Taylor RS, Sagar VA, Davies EJ, et al. Exercise-based rehabilitation for heart failure. Cochrane Database Syst Rev. 2014;1:4.

5. Flynn KE, Piña IL, Whellan DJ, et al. Effects of exercise training on health status in patients with chronic heart failure: HF-ACTION randomized controlled trial. JAMA. 2009;301:1451-1459. doi:10.1001/ jama.2009.457

6. Gary RA, Cress ME, Higgins MK, et al. A combined aerobic and resistance exercise program improves physical functional performance in patients with heart failure: a pilot study. J Cardiovasc Nurs. 2012;27 (5):418-430. doi:10.1097/JCN.0b013e31822ad3c3

7. Ponikowski P, Voors AA, Anker SD, et al. 2016 ESC Guidelines for the diagnosis and treatment of acute and chronic heart failure: the Task Force for the diagnosis and treatment of acute and chronic heart failure of the European Society of Cardiology (ESC)Developed with the special contribution of the Heart Failure Association (HFA) of the ESC. Eur Heart J. 2016;37:2129-2200.
8. van der Wal MH, van Veldhuisen DJ, Veeger NJ, et al. Compliance with non-pharmacological recommendations and outcome in heart failure patients. Eur Heart J. 2010;31(12):1486-1493. doi:10.1093/ eurheartj/ehq091

9. Nieuwenhuis MM, Jaarsma T, van Veldhuisen DJ, et al. Long-term compliance with nonpharmacologic treatment of patients with heart failure. Am J Cardiol. 2012;110:392-397. doi:10.1016/j.amjcard.20 12.03.039

10. Bandura A. Human agency in social cognitive theory. Am Psych. 1989;44:1175. doi:10.1037/0003-066X.44.9.1175

11. Bandura A, Freeman W, Lightsey R. Self-Efficacy: The Exercise of Control. Springer; 1999.

12. Brawley LR, Rejeski WJ, King AC. Promoting physical activity for older adults: the challenges for changing behavior. Am J Prev Med. 2003;25:172-183. doi:10.1016/S0749-3797(03)00182-X

13. Slovinec D'Angelo ME, Pelletier LG, Reid RD, et al. The roles of self-efficacy and motivation in the prediction of short-and long-term adherence to exercise among patients with coronary heart disease. Health Psych. 2014;33:1344. doi:10.1037/hea0000094

14. Ha FJ, Hare DL, Cameron JD, et al. Heart failure and exercise: a narrative review of the role of self-efficacy. Heart Lung Circ. 2018;27:22-27. doi:10.1016/j.hlc.2017.08.012

15. Klompstra L, Jaarsma T, Stromberg A. Self-efficacy mediates the relationship between motivation and physical activity in patients with heart failure. $J$ Cardiovasc Nurs. 2018;33:211-216. doi:10.1097/JCN.0000000000000456

16. Buck HG, Harkness K, Wion R, et al. Caregivers' contributions to heart failure self-care: a systematic review. Eur J Cardiovascular Nursing. 2015;14:79-89. doi:10.1177/1474515113518434

17. Shuval K, Leonard T, Drope J, et al. Physical activity counseling in primary care: insights from public health and behavioral economics. Ca Cancer J Clin. 2017;67(3):233-244. doi:10.3322/caac.21394

18. Piepoli MF, Binno S, Corrà U, et al. ExtraHF survey: the first European survey on implementation of exercise training in heart failure patients. Eur $J$ Heart Fail. 2015;17(6):631-638. doi:10.1002/ejhf.271

19. Conraads VM, Deaton C, Piotrowicz E, et al. Adherence of heart failure patients to exercise: barriers and possible solutions: a position statement of the Study Group on Exercise Training in Heart Failure of the Heart Failure Association of the European Society of Cardiology. Eur J Heart Fail. 2012;14:451-458. doi:10.1093/eurjhf/hfs048

20. Tierney S, Mamas M, Skelton D, et al. What can we learn from patients with heart failure about exercise adherence? A systematic review of qualitative papers. Health Psychology. 2011;30:401. doi:10.1037/a0022848

21. Marzolini S, Mertens DJ, Oh PI, et al. Self-reported compliance to home-based resistance training in cardiac patients. Eur $J$ Cardiovasc Prev Rehabil. 2010;17(1):35-49. doi:10.1097/HJR.0b013e32 $832 \mathrm{da} 020$

22. Klompstra L, Jaarsma T, Stromberg A. Physical activity in patients with heart failure: barriers and motivations with special focus on sex differences. Patient Prefer Adherence. 2015;9:1603-1610. doi:10.2147/PPA.S90942

23. Deci EL, Ryan RM. The empirical exploration of intrinsic motivational. Adv Exp Soc Psychol. 1964;13:39.

24. McAuley E, Wraith S, Duncan TE. Self-efficacy, perceptions of success, and intrinsic motivation for exercise. J Appl Soc Psychol. 1991;21(2):139-155. doi:10.1111/j.1559-1816.1991.tb00493.x

25. Jaarsma T, Klompstra L, Ben Gal T, et al. Increasing exercise capacity and quality of life of patients with heart failure through Wii gaming: the rationale, design and methodology of the HF-Wii study; a multicentre randomized controlled trial. Eur J Heart Fail. 2015;17 (7):743-748. doi:10.1002/ejhf.305 
26. Stenstrom $\mathrm{CH}$, Boestad C, Carlsson M, et al. Why exercise?: a preliminary investigation of an exercise motivation index among individuals with rheumatic conditions and healthy individuals. Physiother Res Int. 1997;2(1):7-16. doi:10.1002/pri.75

27. Dzewaltowski D. Toward a model of exercise motivation. J Sport Exerc Psychol. 1989;11:251-269. doi:10.1123/jsep.11.3.251

28. Zigmond AS, Snaith RP. The hospital anxiety and depression scale. Acta Psychiatr Scand. 1983;67:361-370. doi:10.1111/j.16000447.1983.tb09716.x

29. Broman J-E, Smedje H, Mallon L, et al. The Minimal Insomnia Symptom Scale (MISS). Upsala J Med Sci. 2008;113(2):131-142. doi:10.3109/2000-1967-221

30. Nasreddine ZS, Phillips NA, Bedirian V, et al. The Montreal Cognitive Assessment, MoCA: a brief screening tool for mild cognitive impairment. J Am Geriatr Soc. 2005;53:695-699. doi:10.1111/ j.1532-5415.2005.53221.x

31. Rector T. Patient's self-assessment of their congestive heart failure: II. Content, reli-ability and validity of a new measure-The Minnesota Living with Heart Failure Questionnaire. Heart Fail. 1987;3:198.

32. Cantril H. The Pattern of Human Concerns. New Brunswick, NJ: Rutgers University Press; 1965.

33. Rajati F, Sadeghi M, Feizi A, et al. Self-efficacy strategies to improve exercise in patients with heart failure: a systematic review. ARYA Atheroscler. 2014;10:319.

34. van der Wal MH, Jaarsma T. Adherence in heart failure in the elderly: problem and possible solutions. Int J Cardiol. 2008;125(2):203-208. doi:10.1016/j.ijcard.2007.10.011

35. Herring MP, Puetz TW, O’Connor PJ, et al. Effect of exercise training on depressive symptoms among patients with a chronic illness: a systematic review and meta-analysis of randomized controlled trials. Arch Intern Med. 2012;172(2):101-111. doi:10.1001/ archinternmed.2011.696

36. von Haehling S, Arzt M, Doehner W, et al. Improving exercise capacity and quality of life using non-invasive heart failure treatments: evidence from clinical trials. Eur J Heart Fail. 2020;23 (1):92-113. doi:10.1002/ejhf.1838

37. Fivecoat HC, Sayers SL, Riegel B. Social support predicts self-care confidence in patients with heart failure. Eur $J$ Cardiovasc Nurs. 2018;17:598-604. doi:10.1177/1474515118762800
38. Riegel B, Lee CS, Albert N, et al. From novice to expert: confidence and activity status determine heart failure self-care performance. Nurs Res. 2011;60:132-138. doi:10.1097/NNR.0b013e31820978ec

39. Klompstra L, Liljeroos M, Jaarsma T, et al. Experience of physical activity described by patients with heart failure who have received individualized exercise advice: a qualitative study. J Rehabil Med. 2021;53(1):584. doi:10.2340/16501977-2771

40. Izawa KP, Watanabe S, Oka K, et al. Age-related differences in physiologic and psychosocial outcomes after cardiac rehabilitation. Am J Phys Med Rehabil. 2010;89(1):24-33. doi:10.1097/ PHM.0b013e3181c5607d

41. Warehime S, Dinkel D, Alonso W, et al. Long-term exercise adherence in patients with heart failure: a qualitative study. Heart Lung. 2020;49(6):696-701. doi:10.1016/j.hrtlng.2020.08.016

42. Crutzen R, Ruiter R. Interest in behaviour change interventions: a conceptual model. Eur Health Psychol. 2015;17:6-11.

43. Knittle K, Nurmi J, Crutzen R, et al. How can interventions increase motivation for physical activity? A systematic review and meta-analysis. Health Psychol Rev. 2018;12(3):211-230. doi:10.1080/17437199.2018.1435299

44. Izawa KP, Watanabe S, Omiya K, et al. Effect of the self-monitoring approach on exercise maintenance during cardiac rehabilitation: a randomized, controlled trial. Am J Phys Med Rehabil. 2005;84 (5):313-321. doi:10.1097/01.PHM.0000156901.95289.09

45. Poli A, Kelfve S, Klompstra L, et al. Prediction of (Non) participation of older people in digital health research: exergame intervention study. J Med Internet Res. 2020;22(6):e17884. doi:10.2196/17884

46. Thompson D, Peacock O, Western M, et al. Multidimensional physical activity: an opportunity not a problem. Exerc Sport Sci Rev. 2015;43(2):67. doi:10.1249/JES.0000000000000039

47. Bäck M, Öberg B, Krevers B. Important aspects in relation to patients' attendance at exercise-based cardiac rehabilitation-facilitators, barriers and physiotherapist's role: a qualitative study. $B M C$ Cardiovasc Disord. 2017;17:1-10. doi:10.1186/s12872-017-0512-7
Patient Preference and Adherence

\section{Publish your work in this journal}

Patient Preference and Adherence is an international, peer-reviewed, open access journal that focusing on the growing importance of patient preference and adherence throughout the therapeutic continuum. Patient satisfaction, acceptability, quality of life, compliance, persistence and their role in developing new therapeutic modalities and compounds to optimize clinical outcomes for existing disease states are major areas of interest for the journal. This journal has been accepted for indexing on PubMed Central. The manuscript management system is completely online and includes a very quick and fair peer-review system, which is all easy to use. Visit http:// www.dovepress.com/testimonials.php to read real quotes from published authors. 